\title{
El cognitivismo: perspectivas pedagógicas, para la enseñanza y aprendizaje del idioma inglés, en comunidades hispanohablantes.
}

\section{Cognitivism: pedagogical perspectives, for teaching and learning of the English language, in Spanish-speaking communities}

\section{Cognitivismo: perspectivas pedagógicas, para o ensino e aprendizagem da língua inglesa, em comunidades de língua espanhola}

\author{
Ethel Altez Ortiz \\ Gladys Denis Mamani Quispe \\ aloret@upeu.edu.pe \\ iD https://orcid.org/0000-0002-0998-9447 \\ Universidad Peruana Unión, Perú \\ gladys.mamani@upeu.edu.pe \\ iD https://orcid.org/0000-0002-4677-8120 \\ Rivaldo Montenegro Chino \\ rivaldo.montenegro@upeu.edu.pe \\ D https://orcid.org/0000-0002-9906-4885 \\ Universidad Peruana Unión \\ Universidad Peruana Unión \\ Isabel Alejandrina Delzo Calderón \\ idelzoc@undac.edu.pe \\ iD https://orcid.org/0000-0001-8217-8870 \\ Universidad Nacional Daniel Alcides Carrión \\ Nancy Trujillo Bravo \\ ntrujillo@undac.edu.pe \\ iD https://orcid.org/0000-0001-9229-6926 \\ Universidad Nacional Daniel Alcides Carrión \\ Marina del Águila Gonzales de del Castillo \\ marina 010454@hotmail.com \\ iD https://orcid.org/0000-0003-2343-4533 \\ Universidad Nacional de La Amazonia \\ Peruana
}

Recibido: 03 de enero de 2021

Aceptado: 06 de junio de 2021

\section{Resumen}

El presente artículo tiene el objetivo de analizar las perspectivas pedagógicas del cognitivismo, para orientar el proceso enseñanza aprendizaje del idioma inglés; en este sentido, se desarrollan algunos conceptos y reflexiones sobre el cognitivismo, la enseñanza y el aprendizaje del idioma inglés, cuyos beneficios son traslados a los docentes y estudiantes de lingüística e inglés, quienes tienen marcado protagonismo; los primeros en la condición de facilitadores; los segundos constituidos en los protagonistas de su propio aprendizaje del idioma inglés. En conclusión, el cognitivismo resulta de gran 
aportación para el aprendizaje del idioma inglés, en cuyo proceso se percibe la presencia de los aspectos mentales, internos de los sujetos aprendices.

Palabras clave: Cognitivismo, aprendizaje, enseñanza, idioma inglés.

\begin{abstract}
The present article has the objective of analyzing the pedagogical perspectives of cognitivism, to guide the teaching-learning process of the English language; In this sense, some concepts and reflections on cognitivism, teaching and learning of the English language are developed, the benefits of which are transferred to teachers and students of linguistics and English, who have a marked role; the first in the condition of facilitators; the latter constituted the protagonists of their own learning of the English language. In conclusion, cognitivism is a great contribution to learning the English language, in the process of which the presence of mental aspects, internal to the learning subjects, is perceived.
\end{abstract}

Keywords: Cognitivism, Learning, Teaching, English language.

\title{
Resumo
}

O presente artigo tem como objetivo analisar as perspectivas pedagógicas do cognitivismo, para orientar o processo ensino-aprendizagem da língua inglesa; nesse sentido, são desenvolvidos alguns conceitos e reflexões sobre cognitivismo, ensino e aprendizagem da língua inglesa, cujos benefícios são transferidos para professores e alunos de lingüística e inglês, que têm um papel marcante; o primeiro na condição de facilitadores; estes últimos constituíram os protagonistas de seu próprio aprendizado da língua inglesa. Concluindo, o cognitivismo é de grande contribuição para a aprendizagem da língua inglesa, processo em que se percebe a presença dos aspectos mentais, internos dos sujeitos aprendizes.

Palavras-chave: Cognitivismo, aprendizagem, ensino, língua inglesa.

\section{Introducción}

A fines de los 50s, los modelos conductivistas se quedan solos, quedando cognitivos; es decir, los modelos que derivan de las ciencias cognitivas, cuyos defensores se ubicaron en una abierta oposición contra el conductismo (Ertmer y Newby, 1993). Cuando se observa el universo educativo, cultural y artístico, se quedan, entre los ojos, los conflictos diversos, las discusiones académicas; en este espacio tiene la presencia del cognitivismo, para precisar si arte o conocimiento (Castro, 2005). El 
cognitivismo es un término empleado en demasía en oposición al conductismo, entre los años 1955 y 1965, cuya transición se dio en forma lenta y progresiva, percibiendo que las condiciones favorecían el "surgimiento y la expansión de la ciencia cognitiva". Este panorama significó la presencia de las teorías de aprendizaje, las cuales recurrieron a la existencia, la generación y el ensayo de otros modelos; es decir, aparecen en escenario los nuevos paradigmas, los modelos y los enfoques, posiblemente mucho más complejos, todos provenientes de las ciencias cognitivas, dejando el entendido de que el cognitivismo es una teoría basada en concepciones y paradigmas cognitivistas. Con el cognitivismo, llega la ciencia cognitiva (Zumalabe, 2012).

Martínez (1992), por su parte, aprecia que la investigación interdisciplinaria facilita el estudio de la cognición en seres humanos, cuyo proceso de aprendizaje revela la presencia de varias disciplinas; por ejemplo, psicología, filosofía, inteligencia artificial, neurolingüística, antropología y neurociencias. En este sentido, el cognitivismo se constituye en un enfoque, cuya base son los paradigmas, cuyo escenario es un universo lleno de concepciones convincentes, insuficiencias teóricas y epistemológicas del conductismo. El cognitivismo tiene relación directa con el mentalismo, pues describe los procesos mentales, los procesos internos, procesos cognoscitivos o de conocimiento, en la condición de portadores de información; por entonces, interesa más "la cognición, el aprendizaje como adquisición de conocimientos, la memoria o la conciencia". Así se asigna una denominación: "una ciencia objetiva de la mente".

Fonseca y Bencomo (2011) realiza una revisión documental y un reencuentro histórico, con el propósito de abordar la enseñanza, el aprendizaje, las teorías respectivas y los beneficios para la educación, permitiendo ejercer el análisis de los escenarios, las situaciones, los eventos, también los fenómenos y los comportamientos sociales (a nivel de las personas y de los conjuntos), razón por la cual se hace complejo el abordaje del aprendizaje.

En el escenario educativo y psicológico se observa la presencia significativa de Piaget, precisamente durante el siglo XX, para quedarse por siempre en la mente de los psicólogos y los educadores, generando una relevancia para la psicología, la pedagogía, la epistemología, la lógica y la matemática. Piaget percibe que el desarrollo intelectual no es sino la restauración del conocimiento, elaborando nuevas ideas, nuevos esquemas, 
motivados por cambios externos; el conocimiento se establece con el "equilibrio ente la reflexión teórica y la investigación empírica” (Saldarriaga, Bravo y Loor, 2016).

La enseñanza y el aprendizaje no ha podido liberarse de la Taxonomía de Bloom (Caeiro, 2019). Para Álvarez (2017), la presencia del fenómeno de la cognición significa la "co-dependencia con el organismo viviente", significa "manipular representaciones simbólicas, es acción corporizada guiada perceptualmente, la cual va creando un mundo con sentido para el organismo viviente, en base a dicha relación de co-dependencia" (p. 5). La presencia de las psicologías: conductual, cognitiva e histórico-cultural, es determinante de las teorías de los aprendizajes personales y colectivos, por su puesto también generando distancias y la presencia de ciertas problemáticas (González, 2020). Actualmente, se percibe "la visión representacional de la cognición contrapuesta a un enfoque enactivo de la ciencia cognitiva, que defiende que el conocimiento se origina de la afectación del cuerpo por el mundo ambiente" (Reyes y Roque, 2020, p. 257)

El objetivo del estudio es analizar las perspectivas pedagógicas del cognitivismo, para orientar, de una manera más eficiente y eficaz, el proceso enseñanza aprendizaje del idioma inglés, con el propósito de ayer a los docentes y a los estudiantes, quienes se hallan siempre en un escenario denominado: aula.

\section{Cognitivismo}

\section{Conceptos}

El cognitivismo significó “surgimiento y la expansión de la ciencia cognitiva”, el reclamo de la presencia de las teorías de aprendizaje; es una teoría, cuya base la constituyen las concepciones y paradigmas cognitivistas (Zumalabe, 2012).

Martínez (1992), por su parte, aprecia que el cognitivismo es el estudio de la cognición humana, mediante la recurrencia a varias disciplinas: psicología, filosofía, inteligencia artificial, neurolingüística, antropología y neurociencias; el cognitivismo es un enfoque sostenido sobre paradigmas; tiene una relación directa con el mentalismo, los procesos mentales, los procesos internos, procesos cognoscitivos o de conocimiento; aparece la denominación: "una ciencia objetiva de la mente".

Por otro lado, Leiva (2014) designa que el cognitivismo es "teoría o enfoque del aprendizaje", encargado de estudiar el proceso de aprendizaje del ser humano, relaciona 
el cognitivismo con el conductismo metodológico, sobre la base de teorías semejantes al cognitivismo.

\section{Cognitivismo, enseñanza y aprendizaje}

El aprendizaje es un ámbito complejo, en el cual aparecen diversas teorías; por ejemplo, teorías psicológicas y filosóficas; no quedan al margen, los modelos educativos, las estrategias pedagógicas (Fonseca and Bencomo, 2011). En este sentido, las teorías del aprendizaje responden a la apreciación y las notables coincidencia de los filósofos, los psicólogos, los pedagogos, entre otros; así ven la luz los conductistas, cognitivistas, constructivistas y los conectivistas.

Por su parte, Ertmer y Newby (1993), por entonces, comparten la idea de familiarizar a los docentes con el aprendizaje, desde las perspectivas del conductismo, cognitivismo y constructivismo, cuyos aprendizajes respondan a las teorías, las estrategias, las técnicas, permitiendo la construcción de estructuración, la planificación y la realización de los aprendizajes y sus procesos respectivos. Para quienes, el aprendizaje significa un Cambio de la conducta y de la capacidad de comportamiento, resultados de las prácticas y las experiencias. El cognitivismo surgió y se opuso abiertamente a la teoría conductista, educadores y psicólogos dejaron al margen el método conductista, emplearon un método más complejo, concibiendo que el hombre tiene capacidad de pensar, expresar emociones, tomar decisiones y de manifestar sus ideas, totalmente valiosas para el proceso del aprendizaje. Según este enfoque cognitivista, el proceso de aprendizaje y efectividad requieren observar la conducta, también la capacidad de aprender y procesar los asuntos psicológico (recuerdos, conceptos, etc.), en respuesta a la experiencia y la práctica; el cognitivismo estudia el ambiente, la reacción ante ambiente, mucho interesa cómo interpreta por sí mismo; permite valorar el pensamiento y comportamiento del aprendiz, también los procesos mentales (interpretación, proceso y almacenamiento). Las teorías del cognitivismo tienen se basan sobre la taxonomía de Bloom.

Piaget (citado por Saldarriaga et al., 2016) afirma que no constituye una solución simple a un problema complejo: desarrollo cognoscitivo; el conocimiento se genera mediante un proceso complejo de construcción, cuyo protagonista es el sujeto, quien revela su interacción de vida con la realidad; el objetivo no es la respuesta; lo importante es cómo se produce el aprendizaje; por otro lado, el aprendizaje significativo, punto clave en el constructivismos, desarrollado por Ausubel, para quien el aprendizaje perdure, quedando en la memoria sensorial, memoria a corto plazo y la memoria a largo plazo, en 
esta última se produce el aprendizaje significativo, previo encuentro de los saberes previos y los nuevos saberes. El desarrollo cognitivo demanda la construcción de esquemas mentales, un proceso de reconstrucción permanente, durante etapas y estadíos, cuyas nuevas apariciones son superiores a la anteriores, revelando cambios cuantitativos y cualitativos.

De acuerdo con Sanchez, Costa, Mañoso, Novillo y Pericacho (2019), el cognitivismo se ha constituido en un nuevo paradigma de aprendizaje, especialmente durante la era digital; comparte la visión global del conectivismo; cognitivismo y conectivismo valoran el papel de los protagonistas, en forma activa, durante la elección de los contenidos, las formas y los procedimientos, generando un significado, singular, único y propio. Por su parte, el conectivismo tiene más relación con la era digital, las TICs, principal herramienta de aprendizaje y enseñanza; se observa una contraposición con el constructivismo; el conectivismo posee aspectos cognitivos y constructivistas. Según el cognitivos, el aprendizaje ha demandado la presencia de un currículo enfocado hacia el estudiante, cuyo papel es protagonista y totalmente activo. La creatividad es un motor caótico para el cognitivismo, genera desorden con el conocimiento adquirido, llevándolo o otros escenarios nuevos, muy complejos, los cuales son ordenados posteriormente en un nivel superior; este proceso es permanente.

\section{Enseñanza del inglés}

Por varios factores, el inglés se ha hecho una lengua universal y totalizadora, no solamente es una necesidad, sino un privilegio, por la fuerza de la globalización y las exigencias de la sociedad actual. La enseñanza del inglés implica una exigencia sustantiva, para la infancia y resto de la vida del hombre; por eso se hace énfasis en la infancia y la niñez, con el objetivo de lograr niños capaces para entender mejor este idioma. La introducción en el inglés se realiza mediante la enseñanza de vocabulario y gramática del idioma; también enseñándoles cómo utilizar los vocabularios y las expresiones gramaticales de manera apropiada, buscando la fluidez en contextos y situaciones de la vida cotidiana. Significa conocer y poseer un "amplio abanico de las metodologías", comparar y contrastar metodologías, generar las soluciones requeridas, también desarrollar opiniones propias respecto de las metodologías. "Para ello es el 
propio centro escolar y el maestro quien debe idear su propia estrategia de acción en el centro y en el aula y seguir unas pautas básicas" (Lareki, 2014, p. 8).

Toda enseñanza implica la presencia y el uso pertinente de materiales y recursos, los cuales deben ser didácticos, representativos, adaptados, visuales, auditivos, audiovisuales, recreativos: objetos reales, flashcards and wordscards, cuentos, comics o revistas, las nuevas tecnologías, sin descuidar la comunicación, la participación, la práctica de las capacidades lingüísticas: listening, speaking, reading, writing, generando interés, motivación, experiencias cercanas, con adaptación al nivel, la edad y las necesidades, mayor número de variables posibles, tipos de agrupamientos, resultados concretos, instrucciones clara y sencillas, buenas conexiones, desarrollo de competencias básicas (Lareki, 2014).

Por otro lado, Lareki (2014) propone la secuencia de algunas actividades: introducción, desarrollo, consolidación, refuerzo, ampliación y evaluación.

\section{Cognitivismo y aprendizaje}

El cognitivismo hizo aportaciones significativas para la comprensión del aprendizaje. En este contexto se plantean cuestiones: ¿cómo el cognitivismo ayuda al aprendizaje?, ¿qué ventajas ofrece el cognitivismo para el aprendizaje? y, por último, ¿qué deficiencias ofrece el cognitivismo para la enseñanza y aprendizaje?

Respondiendo la primera cuestión planteada, la teoría del desarrollo cognitivo de Jean Piaget, principal representante del cognitivismo, es una de las más importantes. Se enfoca en cómo el estudiante conoce el mundo externo mediante los sentidos, siempre en la perspectiva evolutiva del proceso mental del estudiante y sus habilidades cognitivas complejas, construidas por sí mismos y con la gran ayuda del docente y diferenciando las etapas: sensoriomotora, preoperacional, operaciones concretas y de operaciones formales; se construye el conocimiento mediante la interacción con el medio, generando asimilación y acomodación (Saldarriaga et al., 2016).

Teorías del aprendizaje

Otras teorías que aportaron para la enseñanza y del aprendizaje en el cognitivismo son: 1)Teoría del aprendizaje por descubrimiento de Jerome Bruner (1978, citado por Guilar, 2009), la categorización o procesos simplifican la interacción con la realidad, mediante la agrupación de objetos, sucesos o conceptos; el aprendiz construye conocimiento (genera proposiciones, verifica hipótesis, realiza inferencias), cuyas propias categorías son modificadas por su interacción con el ambiente. Bruner afirma que 
estos modos de representación son desarrollados en los niños y niñas, si cambian cognitivamente. La representación inactiva corresponde al período sensorio-motor de Piaget (primer año de vida), la representación icónica es posible si los niños se encuentran en el período preoperatorio (3, 4, 5 años); aproximadamente a los seis años de edad, es posible la representación simbólica, si los niños y niñas son capaces de utilizar ideas abstractas, símbolos lingüísticos y lógicos para entender y representar la realidad. El cognitivismo busca modos de profundizar en un determinado conjunto de conocimientos.

2) Teoría de las inteligencias múltiples de Howard Gardner (1983, citado por Gamandé (2015). Cada ser humano tiene una combinación única de inteligencia; clasificando 8 tipos de inteligencia: inteligencia lingüística, inteligencia lógico-matemático, inteligencia espacial, inteligencia musical, inteligencia corporal y cenestésica, inteligencia interpersonal e inteligencia naturalista; Gardner y sus colaboradores afirman que Stephen Hawking no posee mayor inteligencia que Leo Messi, cada uno ha desarrollado un tipo de inteligencia diferente a la teoría de Gardner; es una metodología más adecuada para la enseñanza, tiene de base el desarrollo de las competencias individuales; la inteligencia es múltiple en cada individuo, depende del entorno social, experiencias y educación. 3) Teoría ecléctica de Robert Gagné (1970, citado por Gottberg, Noguera y Noguera (2012). Se enmarca dentro de las teorías del procesamiento de información, también llamadas teorías cibernéticas. El aprendizaje del individuo es similar al funcionamiento de una computadora, es activo, depende de la información recibida, de cómo se organiza y procesa la información. 4) Teoría histórico-cultural o sociocultural del psiquismo humano de Lev Vygotsky (1996, citado por Martínez, 1999). La actividad mental es humana; es el resultado del aprendizaje social, de la interiorización de la cultura y de las relaciones sociales; el lenguaje es el principal mediador; permite la adquisición de pensamiento abstracto; plantea la teoría del nivel de desarrollo real y el nivel de desarrollo potencial del niño. 5) Teoría del desarrollo psicosocial de Erikson (1968, citado por Martínez, 1999). Se basa sobre la búsqueda del "yo"; propuso el concepto de desarrollo de la personalidad desde la infancia hasta la vejez; en cada una de las nuevas etapas de la vida, la persona ha logrado la competencia vital; la persona experimentará una sensación de dominio; ayuda a resolver las metas durante la siguiente etapa vital.

Cognitivismo, la enseñanza y el aprendizaje del inglés

De acuerdo con Caeiro (2019), en el cognitivismo se da prioridad al estudiante, quien es protagonista; por otro lado, el docente cumple el papel de mediador. En este marco, corresponde plantearse las siguientes interrogantes: ¿El estudiante tiene los 
conocimientos previos acerca del tema a tratar? ¿Cuál es su nivel de aprendizaje? ¿Cómo se da su aprendizaje? Para responder estas preguntas, las teorías del cognitivismo aportan la taxonomía de Bloom, parcialmente cambiada por Anderson y Krathwol, quienes añadieron la "creación” de conocimientos nuevos; la taxonomía de Anderson y Krathwohl comprende los procesos: reproducir o recordar, conceptualizar, aplicar, analizar, evaluar y crear, todo ello mencionado jerárquicamente.

Bancayán (2017) afirma que el docente, desde mediados del siglo pasado, enfoca la planificación, la implementación, la ejecución y la evaluación; nacen las taxonomías de los propios docentes. Entre ellas destaca la de Anderson y Krathwohl. 1) Reproducción: Trata de la retención de memoria que tiene el estudiante, para ello el docente plantea preguntas, en donde el estudiante tendrá que: recordar, definir, contar, describir y reconocer; en relación a los conocimientos previos. 2) Conceptualización: El estudiante debe de ser capaz de integrar los conocimientos nuevos con los conocimientos previos, utilizando sus propias palabras; el docente, por tanto, tiene que mostrar, escuchar, preguntar, comparar, contrastar y examinar; generando en los alumnos las habilidades: parafrasear, explicar e interpretar. 3) Aplicación: El docente debe de plantear tareas para os estudiantes, en donde el aprendiz, pondrá a práctica todo lo aprendido previamente; generando las habilidades: resolución de problemas, demostrar el uso de los conocimientos, calcular, compilar, completar, ilustrar, construir; en virtud, de ser un receptor activo. 4) Análisis: De cierta información, el estudiante debe descomponer la información en distintas partes y buscar la relación entre ellas; esto implica la conceptualización de causa y efecto. El docente cumple la función de: guía, observador, evaluador y actúa como un recurso, pregunta y organiza; mientras que el estudiante desarrolla las siguientes habilidades: explicación, descubrimiento, argumentación, debate, piensa profundamente, prueba, examina, pregunta, calcula, investiga y consulta. 5) Evaluación: El estudiante debe realizar juicios propios, basados en criterios (calidad, efectividad, eficiencia y consistencia) y normas, de acuerdo con los resultados de un análisis. El docente cumple la labor importante de guiar y aclarar. 6) Crear: "Aunque demanda pensamiento creativo al estudiante, no es completamente una expresión creativa libre sin los límites que impone la tarea de aprendizaje o la situación" (p. 116). De alguna manera aún dependen del docente.

En el contexto del cognitivismo, el aprendizaje de los estudiantes se produce mediante el proceso de información interno e interacción con el ambiente. El aprendizaje de una lengua extranjera requerirá aprender las formas de la lengua y las estructuras 
conceptuales asociadas. Las conceptualizaciones simbolizadas en la lengua extranjera no serán iguales en su forma, a las de la lengua materna, en los niveles: léxico, semántico (Martín, 2001). El enfoque cognitivo permite abordar las diferencias formales y las conceptuales; según las teorías cognitivistas, el aprendizaje se logra mediante la obtención de conocimientos, gracias a la interrelación del profesor y del alumno, en el aula. En el siglo XIX, se vigoriza la generalización de que los estudiantes construyen aprendizajes significativos, mediante la jerga en el contexto social, al deliberar y razonar sobre el lenguaje mismo. La perspectiva del lenguaje pasa, de ser social e interactiva, a una perspectiva cognitiva: desarrollo de capacidades cognitivas mejoradas. Para Cummins (2011, citado por Aljurre, Arcienegas y Castillo, 2014), se generan las ventajas cognitivas del aprendizaje de dos o más lenguas, mostrando transferencia en el cerebro durante el proceso de aprendizaje, si su enseñanza es apropiada y a la edad y desarrollo cognitivo de los alumnos: atención, memoria, percepción, reconocimiento y uso del lenguaje en el proceso del aprendizaje.

Aprendizaje por descubrimiento. Las enseñanzas recibidas son reconstruidas por el alumno al asimilar un modelo, antes de ser aprendidas e incorporadas significativamente durante el proceso de su composición cognitiva. El bilingüismo implica la capacidad de hablar correctamente dos lenguas: materna y extranjera. Es necesario el desarrollo de capacidades en la acción y en lo teórico, poniendo énfasis en el segundo. La mayoría de los estudios muestran cómo los estudiantes bilingües tienen un mayor conocimiento explícito sobre la estructura y las funciones del lenguaje (Aljure, Arciniegas y Castillo, 2014).

Aprendizaje significativo. Ausubel plantea la teoría de aprendizaje significativo, con el propósito de entender que significa incorporar nuevos conocimientos a los ya obtenidos; este aprendizaje, en el alumno del inglés, se logra mediante tres tipos de aprendizaje significativo: 1) Aprendizaje de representaciones: el niño primero adquiere el vocabulario, luego lo relaciona con objetos, personas, animales y entorno social, respetando los principios básicos de la lingüística cognitiva. Para Martín (2001): El lenguaje está íntimamente relacionado con otros campos de la cognición humana: psicológicos, culturales, sociales, etc. y no puede entenderse adecuadamente sin apelar a criterios de interdisciplinariedad. La competencia lingüística no es autónoma, ni puede 
separarse de las demás capacidades cognitivas y habilidades sociales del individuo. (p.220)

2) Aprendizaje de conceptos: el infante, con incorporación de las experiencias concretas, comprende que la palabra "pelota", la puede usar con otras personas, para referirse a objetos similares en la lengua materna. Por otro lado, Quidel, Del Valle, Arévalo, Ñancucheo y Ortiz (2014) afirman que el aprendizaje del idioma inglés en la niñez o a temprana edad es el momento óptimo, tienen la habilidad para aprender una lengua, por la plasticidad cerebral y la falta de especialización cortical; posteriormente la maduración y la organización del cerebro se hacen más especializadas; la capacidad para aprender un idioma decrece. Se experimenta la atrofia "phonological fossilization", el individuo suma tiempo y edad, afecta la pronunciación de una segunda lengua (inglés). 3) Aprendizaje de proposiciones: el alumno forma frases con dos o más conceptos, asimilándolos e integrándolos en su estructura cognitiva. Se presentan las siguientes formas: diferenciación progresiva, reconciliación integradora y combinación. Para el aprendizaje del idioma inglés, es muy importante que el estudiante tenga la primera aproximación a la lengua extranjera. Para Scrivener (2005, citado por Quidel et al., 2014), "los profesores son la fuente principal de entrada del idioma comprensible para los alumnos, el tiempo que el profesor ocupa en hablar juega una parte importante en la adquisición del lenguaje” (pp. 37 y 38)..

\section{Conclusiones}

Según el cognitivismo, el aprendizaje es activo; el estudiante es el protagonista principal del aprendizaje. Las características son: marcada importancia en los procesos mentales del aprendizaje, los aprendizajes se archivan en la memoria; tienen una base de investigación para la realización de trabajos científicos, las diferentes condiciones ambientales forman parte del proceso de aprendizaje; según la Teoría de Jean Piaget, son incorporados: las explicaciones, los ejemplos y las demostraciones, los cuales constituyen guía para un adecuado aprendizaje; el conocimiento es significativo, ayuda la organización y relación de nueva información con el conocimiento; el sujeto es un procesador activo de la información, mediante el registro y la organización de la información,

El cognitivismo es una teoría adaptada a la realidad educativa del idioma inglés; las desventajas en el ámbito general de aprendizaje: limitación de enseñanza; el estudiante, mediante esquemas, desarrolla su experiencia personal; asimila nuevos conocimientos 
otorgados por el docente y no desarrolla capacidad crítica, fundamental para el aprendizaje del idioma inglés; solamente asimila conceptos básicos; las propuestas cognitivistas son buenas y favorables para el aprendizaje del idioma inglés. El alumno es el principal protagonista y el docente un mediador de información, desarrollando el análisis y la observación.

Aprendizaje y adquisición son dos conceptos diferentes, en el contexto de la enseñanza y el aprendizaje del inglés. El aprendizaje se realiza mediante observación de reglas; la adquisición mediante la fluidez del idioma, de manera inconsciente. El bilingüismo y el cognitivismo hacen que el aprendizaje se convierta en adquisición, de acuerdo con los conceptos de Ausubel: aprendizaje significativo y aprendizaje por descubrimiento.

El estudiante es evaluado de acuerdo con la taxonomía de Anderson y Krathwohl: una secuencia ordenada y lógica, generando en el docente una visión compleja de las habilidades de los estudiantes, mediante acciones concretas en el aula o fuera de la misma; también sobre la utilización de método y estrategias, para una mejor enseñanza.

\section{Referencias}

Aljurre, L. H., Arcienegas, M. C. y Castillo, M. F. (2014). Aprendizaje de lenguas y desarrollo cognitivo de los niños en edad escolar. El Astrolabio, 116-124.

Retrieved from http://astrolabio.phipages.com/ediciones-anteriores/astrolabio_132/articulo 9

Álvarez, J. (2017). El enactivismo como superación teórica de la visión dualista y abstracta del cognitivismo. [Tesis, Universidad Alberto Hurtado]. Retrieved from https://repositorio.uahurtado.cl/bitstream/handle/11242/23777/FILAlvarezH.pdf?se quence $=1$

Bancayán, C. (2017). Operacionalización de la taxonomía de Anderson y Krathwohl para la docencia universitaria. Paideia XXI, 3(4), 109-119.

https://doi.org/10.31381/paideia.v3i4.931

Caeiro, M. (2019). Recreando la texonomía de Bloom para niños artistas. Artseduca, (24), 66-84. https://doi.org/10.6035/Artseduca.2019.24.6

Castro, S. J. (2005). En defensa del cognitivismo en el arte. Revista de Filosofia, 30(1), 
147-164. https://doi.org/10.5209/rev_RESF.2005.v30.n1.10417

Ertmer, P. A. y Newby, T. J. (1993). Conductismo, cognitivismo y constructivismo: una comparación de los aspectos críticos desde la perspectiva del diseño de instrucción. Performance Improvement Quarterly, 6(4), 50-72. Retrieved from http://www.aprendiendoenlinea.com

Fonseca, H. y Bencomo, M. (2011). Teorías del aprendizaje y modelos educativos: revisión histórica. Salud, Arte y Cuidado, 4(Suplemento 1), 71-93. Retrieved from https://dialnet.unirioja.es/servlet/articulo?codigo $=3938580$

Gamandé, N. (2015). Las inteligencias múltiples de Howard Gardner: Unidad piloto para propuesta de cambio metodológico. [Tesis, Universidad Internacional de la Rioja]. https://doi.org/10.1103/PhysRevB.64.092503

González, E. (2020). Psicologías conductual y cognitiva: un prototipo de análisis filosófico. Revista Electrónica de Psicología Iztacala, 23(4), 1444-1460. Retrieved from http://www.revistas.unam.mx/index.php/repi/article/view/77715

Gottberg, E., Noguera, G. y Noguera, M. (2012). El aprendizaje visto desde la perspectiva electrica de Robert Gagne y el uso de las nuevas tecnologias en educacion superior. Revista de Udual México, (53), 50-56. Retrieved from https://www.redalyc.org/pdf/373/37331092005.pdf

Guilar, M. E. (2009). Las ideas de Bruner: "de la revolución cognitiva" a la "revolución cultural.” EDUCERE Ideas Personajes, (44), 235-241. Retrieved from http://ve.scielo.org/pdf/edu/v13n44/art28.pdf

Lareki, I. (2014). Metodologías de enseñanza de la lengua inglesa en Educación Primaria. [Tesis, Universidad de Valladolid]. Retrieved from https://www.mendeley.com/catalogue/metodologías-enseñanza-la-lengua-inglesaen-educación-primaria/\%0Ahttps://core.ac.uk/reader/211091920

Leiva, C. (2014). Conductismo, cognitivismo y aprendizaje. Tecnología En Marcha, 18(1), 66-74. Retrieved from https://dialnet.unirioja.es/servlet/articulo? codigo $=4835877$ 
Martín, M. (2001). El enfoque cognitivo en la enseñanza de idiomas. Didáctica. Lengua y Literatura, 13(13), 217-217. https://doi.org/10.5209/DIDA.20476

Martínez, M. A. (1999). El enfoque sociocultural en el estudio del desarrollo y la educación. Revista Electrónica de Investigación Educativa, 1(1), 1-21. Retrieved from https://redie.uabc.mx/redie/article/view/6/9

Martínez, P. (1992). Procesos mentales y cognitivismo. Revista de Filosofia, V(7), 143160. https://doi.org/10.5209/rev_RESF.1992.v7.12679

Quidel, D., Del Valle, J., Arévalo, L., Ñancucheo, C. y Ortiz, R. (2014). La enseñanza del idioma inglés a temprana edad: su impacto en el aprendizaje de los estudiantes de escuelas públicas. Vivat Academia, (129), 34-56.

https://doi.org/10.15178/va.2014.129.34-56

Reyes, C. A. y Roque, J. (2020). Ciencias cognitivas e neuroética. Revista Bioética, 28(2), 257-264. https://doi.org/10.1590/1983-80422020282387

Saldarriaga, J., Bravo, R. y Loor, R. (2016). La teoría constructivista de Jean Piaget y su significación para la pedagogía contemporánea. Revista Científica Dominio de Las Ciencias, 2(esp.), 127-137. http://dx.doi.org/10.23857/dc.v2i3\%20Especial.298

Sanchez, R., Costa, Ó., Mañoso, L., Novillo, M. Á. y Pericacho, F. J. (2019). Orígenes del conectivismo como nuevo paradigma del aprendizaje en la era digital.

Educación y Humanismo, 21(36), 113-136.

https://doi.org/10.17081/eduhum.21.36.3265

Zumalabe, J. M. (2012). La transición del conductismo al cognitivismo. EduPsykhé, 11(1), 89-111. Retrieved from http://repositorio.ucjc.edu/bitstream/handle/20.500.12020/55/C00044059.pdf?sequ $\underline{\text { ence }=1}$ 